\title{
Phase field simulation of dendrite growth with boundary heat flux
}

\author{
Lifei Du* and Rong Zhang
}

\author{
* Correspondence: \\ dulifei@mail.nwpu.edu.cn \\ Key Laboratory of Space Applied \\ Physics and Chemistry-Ministry of \\ Education, School of Science, \\ Northwestern Polytechnical \\ University, Xi'an 710072, China
}

\begin{abstract}
Boundary heat flux has a significant effect on solidification behavior and microstructure formation, for it can directly affect the interfacial heat flux and cooling rate during phase transition. In this study, a phase field model for non-isothermal solidification in binary alloys is employed to simulate the free dendrite growth in undercooled melts with induced boundary heat flux, and an anti-trapping current is introduced to suppress the solute trapping due to the larger interface width used in simulations than a real solidifying material. The effect of heat flux input/extraction from different boundaries was studied first. With heat input from boundaries, the temperature can be raised and the dendritic morphology changed with gradient temperature distribution caused by the heat flux input coupling with latent heat release during the liquid-solid phase transition. Also, the concentration distribution can be also influenced by this irregular temperature distribution. Heat flux extraction from the boundaries can decrease the temperature, which results in rapid solidification with small solute segregation and concentration changes in the dendrite structures. Also, dendrite growth manner changes caused by undercooling variation, the result of competition between heat flux and latent heat release from phase transition, are also studied. Results indicate that heat flux in the simulation zone significantly reduces the undercooling, thus slowing down the dendrite formation and enhancing the solute segregation, while large heat extraction can enlarge the undercooling and lead to rapid solidification with large dendrite tip speed and small secondary dendrite arm spacing, while solute segregation tends to be steady. Therefore, the boundary heat flux coupling with the latent heat release from the solidification has an effective influence on the temperature gradient distribution within the simulation zone, which leads to the morphology and concentration changes in the dendritic structure formation.
\end{abstract}

Keywords: Computer simulations; Metals and alloys; Rapid solidification; Microstructure; Diffusion

\section{Background}

The mechanical properties of many materials have a significant relationship with the microstructure formation process [1], but in practice, it is difficult to observe the microstructure formation process with experimental methods, and computer simulations can visually show the phase transition process and provide much more information to calculate many other parameters that are related to the mechanical properties with the data achieved from simulations. For decades, the phase field method has become a popular technique to model various types of complex microstructure changes

\section{Springer}

(c) 2014 Du and Zhang; licensee Springer. This is an Open Access article distributed under the terms of the Creative Commons Attribution License (http://creativecommons.org/licenses/by/4.0), which permits unrestricted use, distribution, and reproduction in any medium, provided the original work is properly cited. 
qualitatively, such as solidification, spinodal decomposition, Ostwald ripening, crystal growth and recrystallization, domain micro-structure evolutions in ferroelectric materials, martensitic transformation, dislocation dynamics, and crack propagation [2,3]. The phase field model has been used for computing solidification morphologies to avoid the explicit boundary tracking needed to solve the classical sharp-interface model. With this advantage, phase field methods have attracted considerable interest in the last decades as a means of simulating the solidification process. In phase field models for solidification process, a variable $\phi(r, t)$, called the phase field order parameter, is introduced to indicate the physical state of the system in time and space. This order parameter $\phi(r, t)$ is assigned the value 0 (or -1) in the bulk solid phase and 1 in the bulk liquid phase, and it changes smoothly between these values over a thin transition layer that plays the role of the classical sharp interface. The governing equations for the growth of a solid phase from a liquid need to be derived from the irreversible thermodynamic law and conservation laws for both mass and energy, and the resulting equations need to be applicable to the entire space being modeled without any discontinuities between the various phases present. It also must be possible to determine the parameters in the governing equations from classical thermodynamic and kinetic quantities.

In the past decades, many researches have studied the solidification process using the phase field method [4,5]. Kobayashi [6] developed a simple phase field model for one-component melt growth including anisotropy and used this model to study the formation of various dendritic patterns. He found that the qualitative relations between the shapes of crystals and some physical parameters and noises gave a crucial influence on the side branch structure of dendrites. The first phase field model for alloys was developed by Wheeler et al. [7,8], called the WBM model. Kim et al. $[9,10]$ presented another model for alloys by adopting the thin-interface limit, which is known as the KKS model. Karma [11] presented a phase field formulation to quantitatively simulate microstructural pattern formation in alloys, and the thin-interface limit of this formulation yielded a much less stringent restriction on the choice of interface thickness than previous formulations and permitted one to eliminate non-equilibrium effects at the interface. Dendrite growth simulations with vanishing solid diffusivity showed that both the interface evolution and the solute profile in the solid were accurately modeled by this approach. Recently, solidifications with forced flow or convection were studied in binary alloys in 2D and 3D [12-16]; solidifications of multi-component and multiphase were also studied using phase field methods [17-19].

As known, the undercooling in the liquid melt has a significant effect on the microstructure forming process and thus affects solute diffusion to change the concentration distributions within the structure. In industry, a cooling/heating system can be applied on the chilling wall during the casting process to control the solidification. So, in this study, we introduce complex boundary conditions in order to present the different cooling/heating conditions to find the relation between the structure forming and these solidification conditions. Experimental and numerical investigations have been presented in the past decades to study the heat transfer problems during solidification [20-24] and find that heat flow can significantly affect the temperature distribution and thus determine the quality of the solidification process. But few studies have been carried out to study the heat flux at the boundaries and its influence on phase transition using phase field methods [25]. Especially, the effect of heat flux on microstructures and 
distributions of temperature and concentration still need to be investigated in detail, for temperature field has a directional influence on the solidification process with undercooling. So, in this study, the boundary heat coupling with latent heat release from solidification is the main factor affecting dendritic structure forming in undercooling melts. Different dendrite patterns are obtained by changing the boundary heat flux at different boundaries and directions. Distributions of the concentration change as the result of temperature change caused by different boundary heat flux couplings with latent heat release are also given and analyzed in detail.

\section{Methods}

\section{Model description and simulation method}

The phase field model for non-isothermal simulation of binary systems [26,27] is implemented to study the flow field effect on the concentration and temperature distributions during dendritic growth. The main governing equations are listed below:

- Phase field equation

$$
\frac{\partial \phi}{\partial t}=M_{\phi} \bar{\varepsilon}^{2}\left[\nabla \cdot\left(\eta^{2} \nabla \phi\right)-\frac{\partial}{\partial x}\left(\eta \eta_{\beta}^{\prime} \frac{\partial \phi}{\partial y}\right)+\frac{\partial}{\partial y}\left(\eta \eta_{\beta}^{\prime} \frac{\partial \phi}{\partial x}\right)\right]-M_{\phi}\left(\left(1-x_{\mathrm{B}}\right) H_{\mathrm{A}}+x_{\mathrm{B}} H_{\mathrm{B}}\right)
$$

- Diffusion equation

$$
\frac{\partial x_{\mathrm{B}}}{\partial t}=\nabla \cdot D\left[\nabla x_{\mathrm{B}}+\frac{V_{m}}{R} x_{\mathrm{B}}\left(1-x_{\mathrm{B}}\right)\left(H_{\mathrm{B}}(\phi, T)-H_{\mathrm{A}}(\phi, T)\right) \nabla \phi+\vec{j}_{a t}\right]
$$

- Temperature equation

$$
c_{p} \frac{\partial T}{\partial t}+30 g(\phi) \Delta \widetilde{H} \dot{\phi}=\nabla \cdot K \nabla T
$$

In this model, the phase field variable $\phi$ varies smoothly between 0 in the solid phase and 1 in the liquid phase as we assumed, $x_{\mathrm{B}}$ is the mole fraction of solute $\mathrm{B}$ in solvent $\mathrm{A}$, and $T$ is the temperature. $\epsilon$ is the coefficient of gradient energy, which is determined by the interfacial energy. The anisotropy is included in the system because the phase change kinetics depends upon the orientation of the interface. Here, we introduce the anisotropy $\varepsilon=\bar{\varepsilon} \eta=\bar{\varepsilon}(1+\gamma \cos \kappa \beta)$, where $\bar{\varepsilon}$ is related to the surface energy $\sigma$ and interface thickness $\lambda, \gamma$ is the magnitude of anisotropy in the surface energy, $\kappa$ specifies the mode number, and the expression $\beta=\arctan ((\partial \phi / \partial y) /(\partial \phi / \partial x))$ gives an approximation of the angle between the interface normal and the orientation of the crystal lattice. $\vec{j}_{a t}$ is the anti-trapping current introduced by Karma [11] to suppress the solute trapping due to the larger interface width used in simulations in order to get a more quantitative prediction.

The formulations included in these governing equations are the following:

$$
H_{\mathrm{A}}(\phi, T)=W_{\mathrm{A}} g^{\prime}(\phi)+30 g(\phi) \Delta H_{\mathrm{A}}\left(\frac{1}{T}-\frac{1}{T_{\mathrm{m}}^{\mathrm{A}}}\right)
$$




$$
\begin{aligned}
& H_{\mathrm{B}}(\phi, T)=W_{\mathrm{B}} g^{\prime}(\phi)+30 g(\phi) \Delta H_{\mathrm{B}}\left(\frac{1}{T}-\frac{1}{T_{\mathrm{m}}^{\mathrm{B}}}\right) \\
& c_{\mathrm{p}}=\left(1-x_{\mathrm{B}}\right) c_{\mathrm{A}}+x_{\mathrm{B}} c_{\mathrm{B}} \\
& \Delta \widetilde{H}=\left(1-x_{\mathrm{B}}\right) \Delta H_{\mathrm{A}}+x_{\mathrm{B}} \Delta H_{\mathrm{B}} \\
& K=\left(1-x_{\mathrm{B}}\right) K_{\mathrm{A}}+x_{\mathrm{B}} K_{\mathrm{B}} \\
& \vec{j}_{a t}=\mathrm{a} \lambda(1-\mathrm{k})\left[\frac{2 \mathrm{x}_{\mathrm{B}}}{1+\mathrm{k}-(1-\mathrm{k}) \phi}\right] \frac{\partial \phi}{\partial \mathrm{t}} \frac{\nabla \phi}{|\nabla \phi|}
\end{aligned}
$$

where $\mathrm{g}(\phi)=\phi^{2}(1-\phi)^{2}$ and $W_{\mathrm{A}}, W_{\mathrm{B}}$ are constants, $T_{\mathrm{m}}^{\mathrm{A}}$ and $T_{\mathrm{m}}^{\mathrm{B}}$ are the melting points of pure $\mathrm{A}$ and pure $\mathrm{B}$, respectively. $\Delta H_{\mathrm{A}}$ and $\Delta H_{\mathrm{B}}$ are the heats of fusion per volume, $c_{\mathrm{A}}$ and $c_{\mathrm{B}}$ are their heat capacities, and $R$ is the gas constant. $p(\phi)=\phi^{3}\left(10-15 \phi+6 \phi^{2}\right)$ is a smoothing function, chosen such that $p^{\prime}(\phi)=30 g(\phi)$. The diffusion coefficient is postulated as a function of the phase field variable, $D=D_{\mathrm{S}}+p(\phi)\left(D_{\mathrm{L}}-D_{\mathrm{S}}\right)$, where $D_{\mathrm{S}}$ and $D_{\mathrm{L}}$ are the classical diffusion coefficients in the solid and liquid, respectively. $a$ in Equation 9 is the anti-trapping coefficient and needs to be adjusted to fit the solid concentration of the sharp-interface solution, and in the present calculations, $a=1 / \sqrt{2}$ [28]. $k=c s / c l$ is the partition coefficient, where $c_{\mathrm{L}}\left(c_{\mathrm{S}}\right)$ is the concentration on the liquid (solid) side of the interface.

Governing equations are solved using the standard finite difference methods with the tridiagonal matrix algorithm (TDMA), and time stepping is by explicit Euler scheme. All simulations are carried out in a $1,200 \Delta x \times 1,200 \Delta y$ simulation box with $\Delta x=\Delta y=0.96 \lambda=4.6 \times 10^{-8} \mathrm{~m}$, and the time step is chosen as $\Delta t=1.0 \times 10^{-8} \mathrm{~s}$. All simulations are carried out for $\mathrm{Ni}-\mathrm{Cu}$ binary alloy, and the physical parameters are listed in Table 1. The initial concentrations of the melt, $x_{0}$, and the initial temperature, $T_{0}$, are chosen to make the whole region initially contain supersaturated $(0.86)$ and undercooled $(\Delta T=20.5 \mathrm{~K})$ melt. Zero-Neumann boundary conditions for $\phi$ and $x_{\mathrm{B}}$ are imposed at all boundaries. The boundaries with different heat fluxes are chosen in the temperature-field calculations, and the boundary heat flux can be introduced as $Q=\lambda_{i}(\partial T / \partial x)$; the density of the heat flux at boundaries is the control parameter, which determines the magnitude and direction of the heat flux.

\section{Results and discussion}

\section{Free dendrite growth in undercooling melt}

Figure 1 gives the morphology, concentration, and temperature distribution maps at $t=2.0 \mathrm{~ms}$ during the free dendritic growth in an undercooled melt without heat flux. Typical dendrite microstructure forms with secondary arms due to the random noise introduced in the simulation. The whole dendritic structure and distributions of temperature and concentration have the approximate rotational symmetry. The primary arms have the largest growth speed and the secondary arms' growth was restrained because of high temperature due to the latent heat release during the liquid-solid phase transition. The solute level between the secondary arms is higher than that inside the solid arm because of solute segregation, and the cells of high concentration formed inside of the dendrite structure because rapid solidification in undercooled melts prevents the 
Table 1 Physical parameters used in the simulations $[7,26,27]$

\begin{tabular}{lc}
\hline Parameter & Value \\
\hline Melting temperature of $\mathrm{Ni}$ & $1,728.0 \mathrm{~K}$ \\
Melting temperature of $\mathrm{Cu}$ & $1,358.0 \mathrm{~K}$ \\
Latent heat of $\mathrm{Ni}$ & $2.35 \times 10^{9} \mathrm{~J} / \mathrm{m}^{3}$ \\
Latent heat of $\mathrm{Cu}$ & $1.728 \times 10^{9} \mathrm{~J} / \mathrm{m}^{3}$ \\
Heat conductivity of $\mathrm{Ni}$ & $84.0 \mathrm{~W} / \mathrm{mK}$ \\
Heat conductivity of Cu & $200.0 \mathrm{~W} / \mathrm{mK}$ \\
Specific heat of Ni & $5.42 \times 10^{6} \mathrm{~J} / \mathrm{m}^{3} \mathrm{~K}$ \\
Specific heat of Cu & $3.96 \times 10^{6} \mathrm{~J} / \mathrm{m}^{3} \mathrm{~K}$ \\
Diffusion coefficient of the liquid phase & $1.0 \times 10^{-9} \mathrm{~m}^{2} / \mathrm{s}$ \\
Diffusion coefficient of the solid phase & $1.0 \times 10^{-13} \mathrm{~m}^{2} / \mathrm{s}$ \\
Mole volume of alloy & $7.42 \times 10^{-6} \mathrm{~m}^{3} / \mathrm{mol}$ \\
Surface energy of Ni & $0.37 \mathrm{~J} / \mathrm{m}^{2}$ \\
Surface energy of Cu & $0.29 \mathrm{~J} / \mathrm{m}^{2}$ \\
Interfacial kinetic coefficient of Ni & $3.3 \times 10^{-3} \mathrm{~m} / \mathrm{sK}$ \\
Interfacial kinetic coefficient of Cu & $3.9 \times 10^{-3} \mathrm{~m} / \mathrm{sK}^{2}$ \\
Interface thickness & $4.9 \times 10^{-8} \mathrm{~m}$ \\
Amplitude of the noise fluctuations & 0.4 \\
Model of anisotropy & 4 \\
Magnitude of anisotropy & 0.04 \\
\hline
\end{tabular}

diffusion of the solute. The low solute level presented in the middle of each dendrite arm is also caused by the rapid phase transition with undercooling.

The concentration and temperature profiles along the vertical lines labeled in Figure $1 \mathrm{~b}$ are plotted in Figure 2. The solute diffusion layer is thinner in front of the primary arm tip than that beside the dendrite arms. The solute segregation is reduced in front of the primary dendrite arm tip compared with that beside the arms because sidebranching increases the temperature beside the primary arms as shown in Figure 2. The temperature distribution profiles indicate that the temperature along the primary arms is unsteady because of the side-branching effect, and latent heat release during the liquid-solid phase transition is the main factor to affect the temperature distribution. The solid phase has higher temperatures because the rapid solidification due to large undercooling prevents the heat diffusion. The temperature gradient formed due to the latent heat release changes the solute segregation and irregular microstructure formation. The solute diffusing inside the solid-liquid interface is affected by the temperature level and thus determines the solute level inside the solid dendrite.

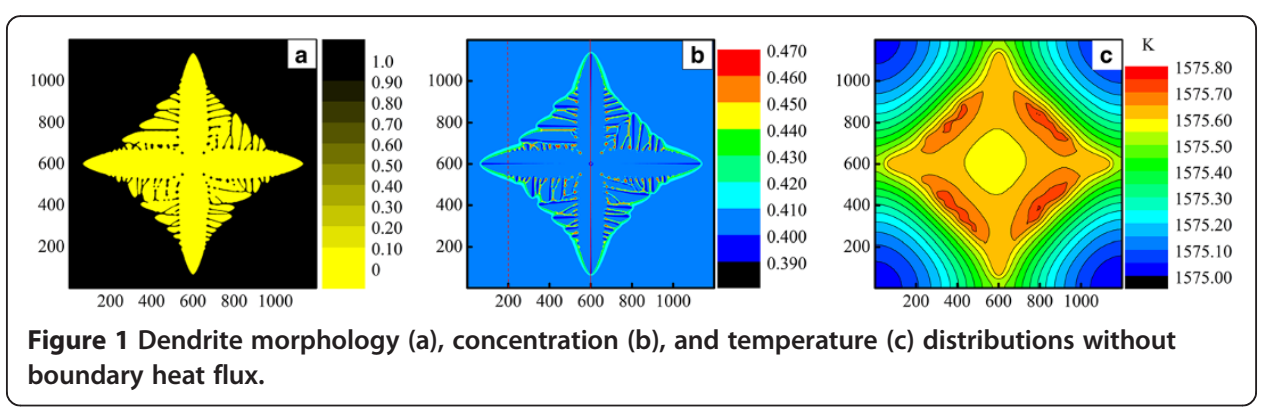



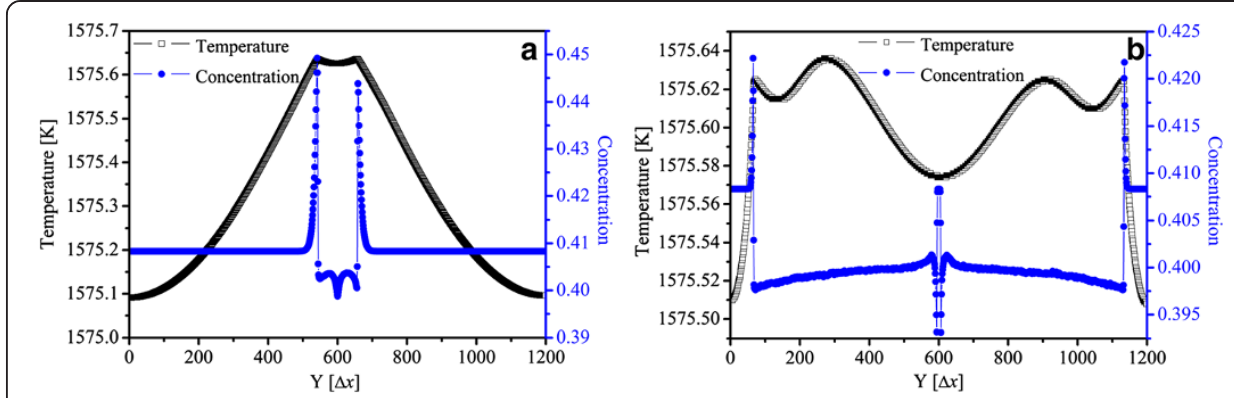

Figure 2 The concentration distribution and corresponding temperature distribution profiles along the vertical lines labeled in Figure 1b. (a) Vertical $X=200$ and (b) central vertical $X=600$.

\section{Dendrite growth with boundary heat flux}

Different heating/cooling conditions during the solidification can significantly change the temperature distributions, thus affecting the solute diffusion and dendrite structure formation. In the following simulations, boundary heat flux is introduced to present different heating/cooling modes during rapid dendrite growth in undercooling melts. Latent heat release during phase transition is also considered in these non-isothermal simulations.

The morphology, concentration, and temperature distribution maps at $t=2.0 \mathrm{~ms}$ during dendritic growth with heat flux input/extraction of $\left|\lambda_{i}\right|=10 \times 10^{-3} \mathrm{~W} / \mathrm{m}^{2}$ from the north (top) boundary are shown in Figure 3. With heat flux input, temperatures near the north boundary are raised, and this prevents dendrite arm growth and sidebranching close to the north boundary. The highest temperature zone finds its position near the primary dendrite arm tip growing towards the north boundary due to the heat flux input coupling with the latent heat release. At the same time, with heat extraction from the north boundary, the primary arm growing towards the north has a high velocity, and sidebranching beside this primary arm is enhanced due to the large undercooling caused by the heat extraction. The length of the primary arm growing towards the north boundary with heat flux input is $12 \%$ less than that with heat flux extraction. The temperature map in Figure 3f shows that the lowest temperature zone

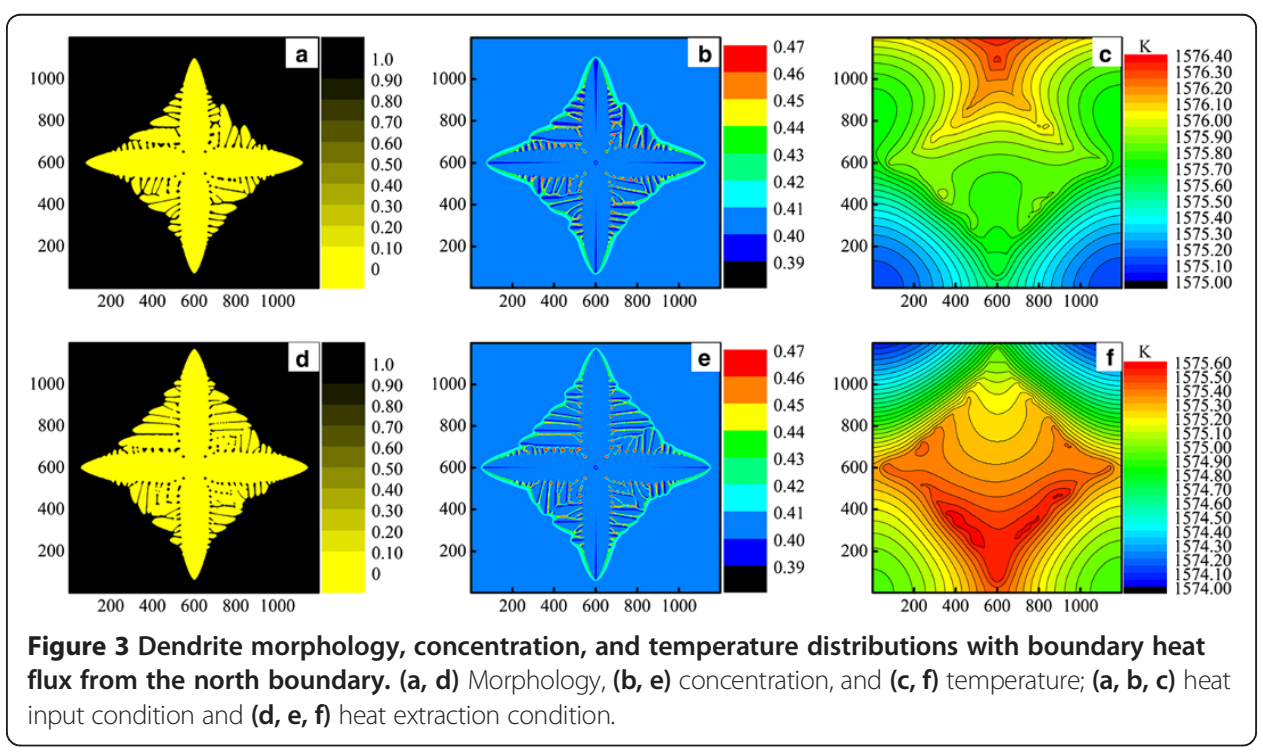


is located at the corners near the north boundary, and the temperate gradient has an opposite direction compared with that of heat input. The concentration distributions have a similar map with heat input and extraction in these two cases, but the solute diffusion is enhanced by high temperature, which shows a thicker solute diffusion layer with slow dendritic structure formation, while large temperature undercooling prevents solute diffusion and leads to an enhanced structure formation with thinner solute diffusion layers.

The concentration distribution and corresponding temperature distribution profiles along the vertical lines are plotted in Figure 4. The solute level along primary dendrite keeps decreasing with the distance from the dendrite center, and this effect is enhanced with the heat flux input from the north boundary. While with the heat flux extraction from the north boundary, an opposite temperature gradient was formed; thus, the solute distribution along the primary arm still keeps decreasing with the temperature rising. That is because the heat flux input from the boundary significantly enlarges the solute segregation near the boundary and leads to an efficient solute diffusion, thus resulting in low solute distribution with high temperature. With heat extraction from the boundary, we find that the solute distribution along the primary dendrite arm near the north boundary keeps steady unlike that without heat flux; this is because the heat extraction partly balances the latent heat release from the liquid-solid phase transition, which keeps the temperature almost unchanged at the solid-liquid interface and makes the solute diffusion approximately steady.

In order to study the complex heat flux effect on the dendrite structure forming process, we simulated the dendrite growing process with heat flux at all boundaries. The morphology, concentration, and temperature distribution maps at $t=2 \mathrm{~ms}$ are shown in Figure 5. With heat input from the south and north boundaries coupling with heat extraction from east and west boundaries, the dendrite primary arms growing in

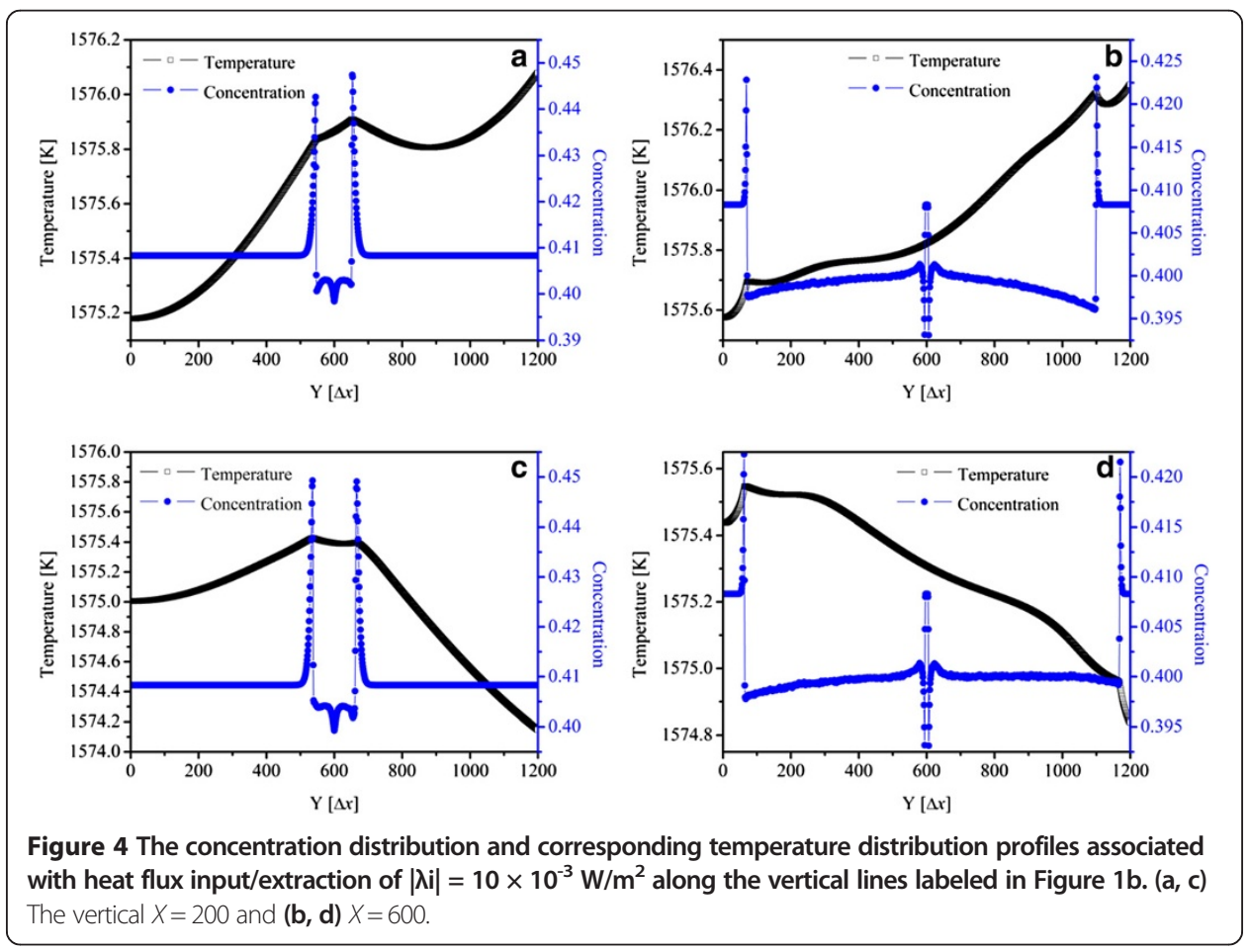




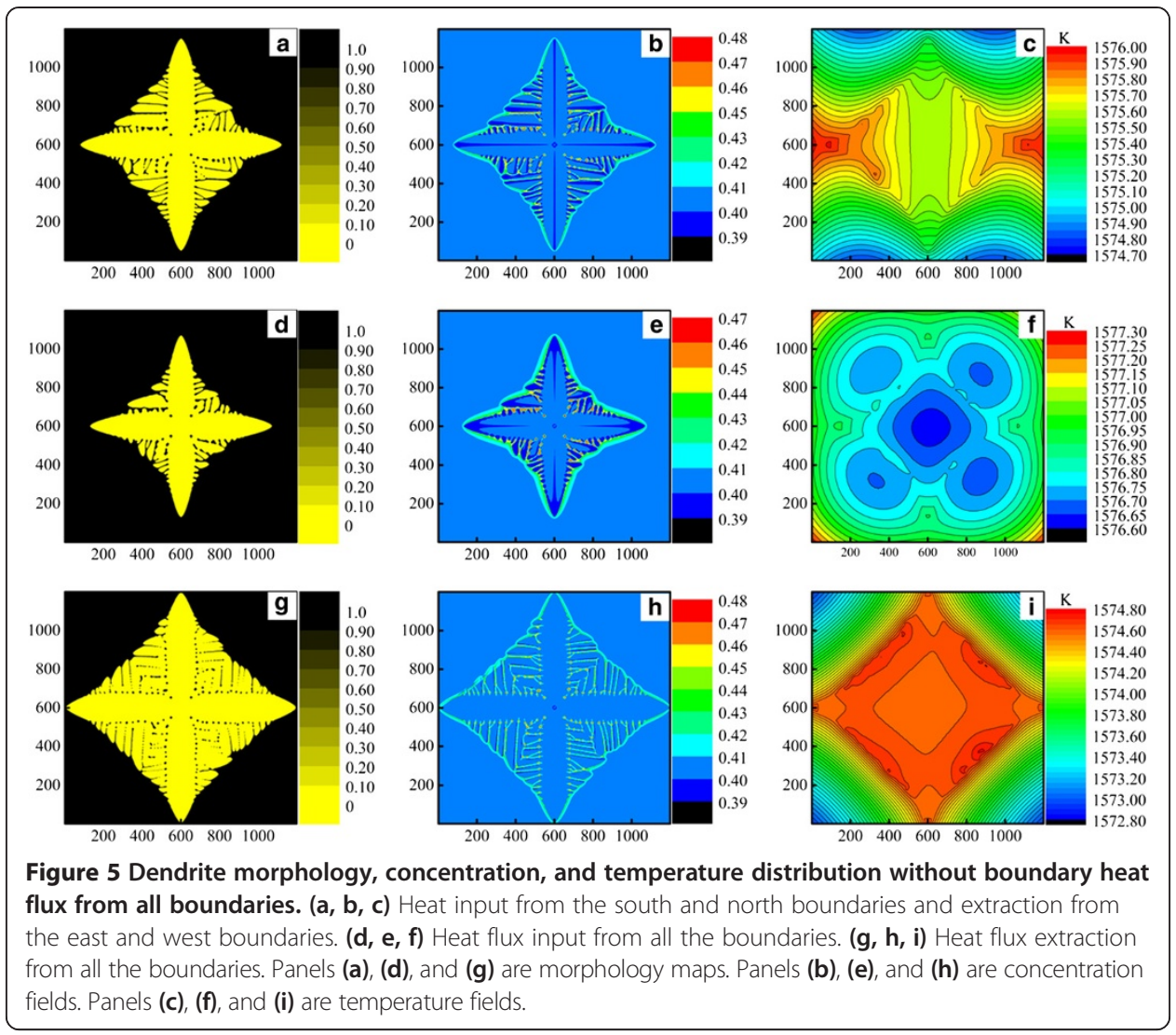

the vertical direction grow fast with much more side branching, while the side branching on the primary arms growing in the horizontal direction is prevented. The length of the primary dendrite arms growing in the horizontal direction is $2.5 \%$ less than that growing in the vertical direction. A large temperature gradient formed between the heat extraction boundaries and solid front, and the temperature has an approximately symmetric distribution both in the vertical and horizontal orientations. Comparing the dendrite pattern formed with heat input/extraction from all boundaries, full dendrite structure can be achieved with heat extraction, and the secondary arms are almost full size and the distance between these arms is small. While with heat input from all boundaries, dendrite growth is suppressed and the number and size of the secondary dendrite arms both decrease. The length of the primary arm growing with heat flux input is $10 \%$ less than that with heat flux extraction. But with high temperature, the solute diffusion is enhanced, and thus, the solute level inside the dendrite is low, especially at the tip of the dendrite arms. But with the large undercooling caused by heat extraction from all boundaries, the solute level inside the solid almost keeps steady with the dendrite arm growth, and the solute diffusion layers is thinner than that with high temperature.

Figure 6 shows the concentration distributions and the corresponding temperature profiles along different vertical lines. The solute segregation beside the primary arms is enlarged by high temperature, and the solute distribution along the center of the primary arm in the vertical direction shows a large difference with different boundary heat conditions. Heat input from the boundary can enhance the solute diffusion and 


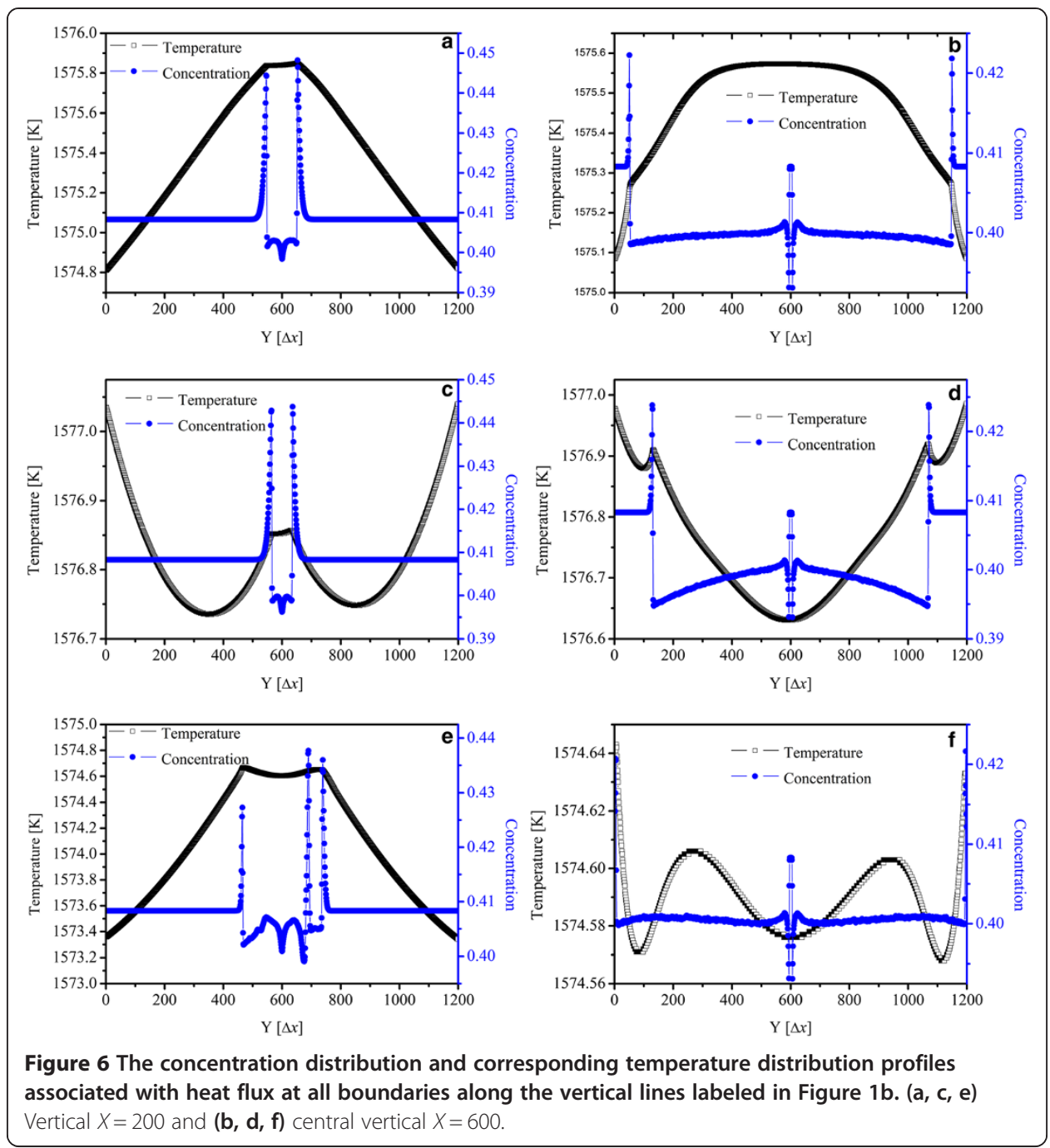

can steadily decrease the solute level with the primary dendrite arm growth while the heat extraction from the boundaries keeps the solute level steady inside the primary arms. High temperature caused by the heat input led to an unsteady solute level, while heat extraction from the boundary makes the solute diffusion steady at the solid-liquid interface region, and thus leads to the uniform solute distribution along the primary arms, which can be considered as a well-distributed phase.

We plotted the temperature distribution profiles along the primary arm growth in the vertical direction at different time steps in Figure 7, and we can see that with heat input, the temperature in front of the dendrite tip keeps increasing. Thus, the dendrite forms at different solute diffusion levels caused by the temperature change, which results in the decreasing solute distribution inside the dendrite arms. While with heat extraction from the boundary, the temperature gradient change with time is small, though the temperature distribution shows an irregular change with time evolution. But at the solid-liquid interface, which is pointed out by arrows in Figure $7 \mathrm{~b}$, the temperature difference is very small; thus, the phase transition almost takes place at the same temperature. So, the solute diffusion keeps a steady level, and this leads to the uniform solute dendrite microstructure. 

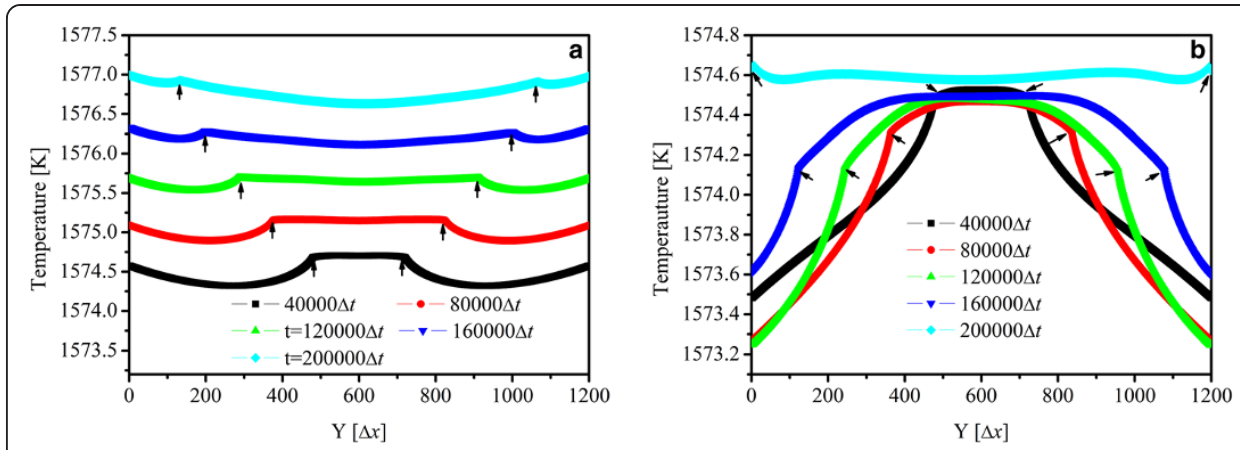

Figure 7 Temperature distribution profiles along the central vertical crossing line at different time steps. Arrows are pointing at the position of the solid-liquid interface. (a) Heat input. (b) Heat extraction.

\section{Effect of different magnitudes of heat flux}

From the 'Dendrite growth with boundary heat flux' section, we can see that the heat flux induced from all boundaries has a significant effect on the heat and solute diffusion as well as the microstructure forming during dendrite growth in undercooled melts. In this section the effect of boundary heat fluxes of different values is investigated. Figure 8 gives the concentration distribution maps with different heat fluxes at the same time. It is clear that with heat extraction, the dendritic structure formation is enhanced, and the more heat extracted, the much more enhanced the structure was. The solute diffusion was suppressed with large undercooling caused by heat extraction, so the solute inside dendritic structures showed a relatively uniform distribution. At the same time, with heat input from all boundaries, the temperature is raised in melts, and high temperature makes the dendrite growth constrained with enhanced solute diffusion. The solute diffusion layer is enlarged with large heat flux at boundaries, which leads to solute diffusion change in the dendritic structure. Especially with large heat input, the dendrite forms with weak side branching, and the primary arms have small growing velocity and tip radius. Figure 9a shows the velocity of the primary dendrite tip at different times; we can see that at the early stage of solidification, all tip growths decrease because of the latent heat release at

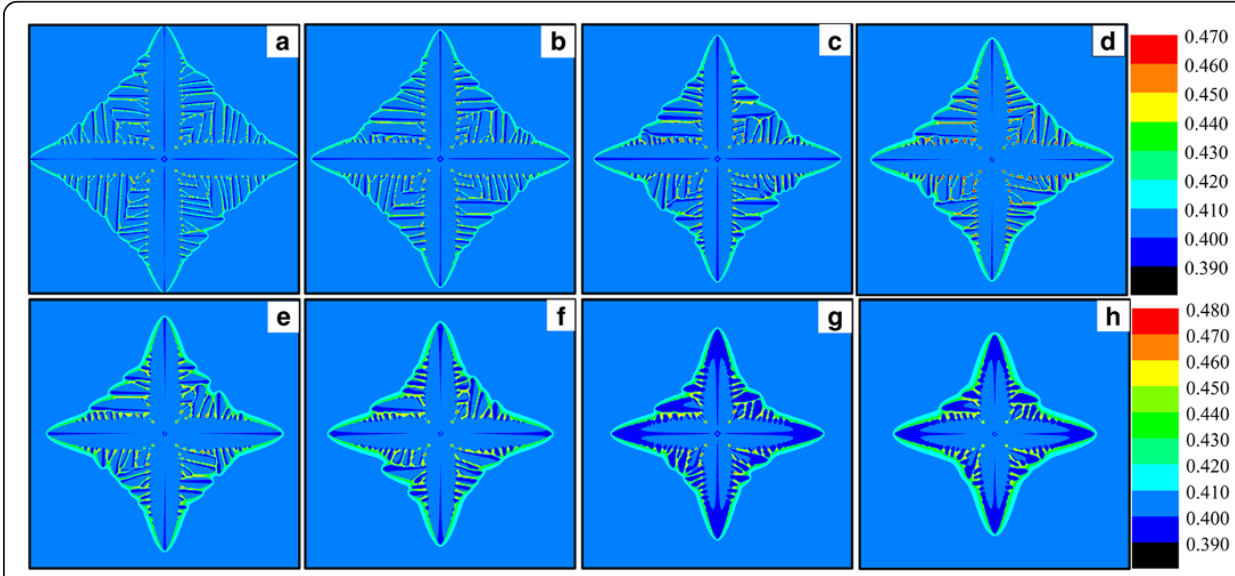

Figure 8 Concentration maps at $\boldsymbol{t}=\mathbf{2} \mathrm{ms}$ with different values of boundary heat flux. (a) $-10 \times 10^{-3} \mathrm{~W} / \mathrm{m}^{2}$, (b) $-6 \times 10^{-3} \mathrm{~W} / \mathrm{m}^{2}$, (c) $-2 \times 10^{-3} \mathrm{~W} / \mathrm{m}^{2}$, (d) 0, (e) $2 \times 10^{-3} \mathrm{~W} / \mathrm{m}^{2}$, (f) $6 \times 10^{-3} \mathrm{~W} / \mathrm{m}^{2}$, (g) $10 \times 10^{-3} \mathrm{~W} / \mathrm{m}^{2}$, and (h) $14 \times 10^{-3} \mathrm{~W} / \mathrm{m}^{2}$. 

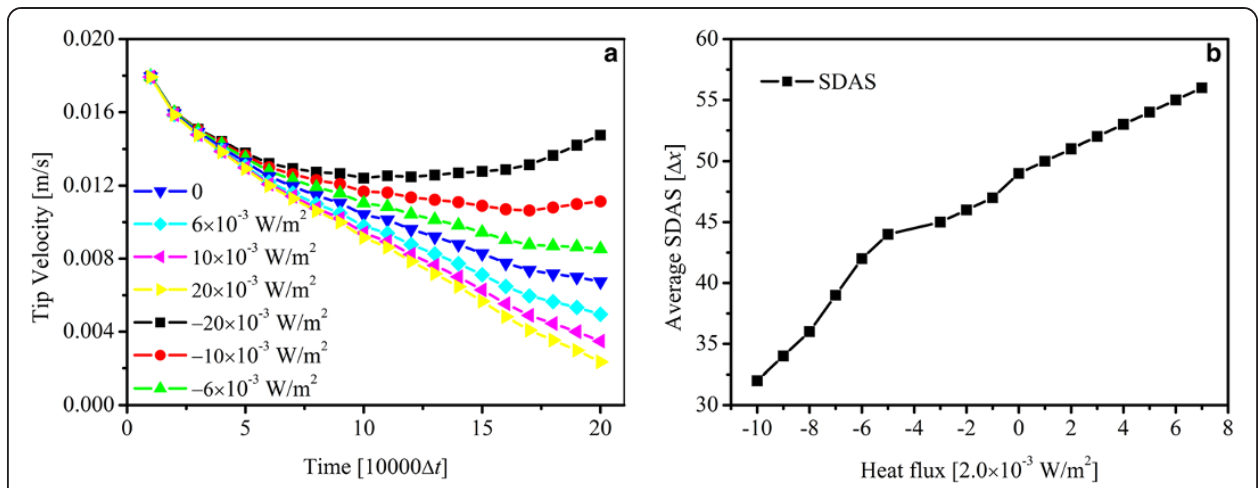

Figure 9 Effect of different values of heat flux. Effect on the velocity of primary dendrite tip (a) and average secondary dendrite arm spacing (SDAS) (b).

the liquid-solid interface. With time evolution, boundary heat flux begins to show its influence on the tip growth: a proper heat extraction from the boundaries can make the tip growth reach a steady speed, and large heat extraction from the boundaries increases the tip growth speed, while heat flux input slows down the tip growth compared with that without heat flux at the boundaries. The average secondary dendrite arm spacing (SDAS) changes with different heat fluxes are plotted in Figure 9b, and the average SDAS keeps increasing with heat flux, changing from large heat extraction to large heat input. A similar effect has been reported during the secondary dendrite arm coarsening in Sn-Bi alloy by means of synchrotron microradiography [29].

In order to understand the relations between temperature and solute segregation inside the interface during phase transition, we plot the temperature and concentration distributions along the central vertical lines at $t=2 \mathrm{~ms}$ with different values of boundary heat flux in Figure 10. The convex in each temperature line indicates the position of the interface since the latent heat released during the phase transition can directly increase the temperature at the interface. The concentration distribution lines plotted in Figure 10b indicate that the solute segregation at the interface is non-equilibrium due to the rapid solidification caused by undercooling. Since the heat diffusion almost takes place in liquid melt in these simulations, the corresponding temperature distribution lines recorded the temperatures at which the phase transition occurred. As we know, latent heat release during phase transitions can raise the temperatures at the
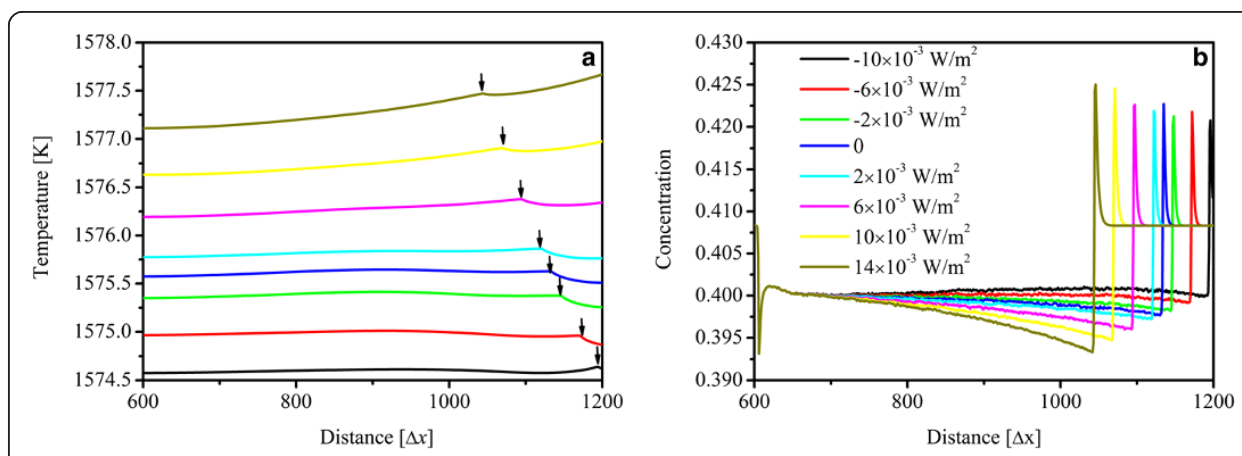

Figure 10 Temperature and concentration distributions along central vertical lines at $2 \mathrm{~ms}$ with different heat flux. (a) Temperature and (b) concentration. 
interface, and this effect is enlarged with time evolution; so, the temperature at which the solidification takes place increases with time, which leads to solute diffusion changes with time. Thus, the concentration distribution inside the dendrite primary arms keeps changing with time, too. With heat flux extracted from the boundaries, the temperature in the melt decreases with time at a certain rate, and this can partly counterbalance the latent heat release, which makes the phase transition at a steady velocity (as shown in Figure 9a) with the unchanged temperature, and this leads to uniform concentration distributions inside dendrite tips, which can be seen with $\lambda_{i}=-10 \times 10^{-3} \mathrm{~W} / \mathrm{m}^{2}$ in Figure 10b. But at the same time, with heat flux input, the temperature is raised in the melt. Coupling with latent heat release, the temperature at which solidification takes place is significantly increased, and solute segregation is kept enhanced with time evolution, which decreases the concentration with time. And this effect could be enlarged with the increasing of heat flux input. Figure 11 gives the maximum and minimum concentration inside the solid-liquid interface near the tip of the primary dendrite arm, and a solute diffusion coefficient was defined as $k \mathrm{c}=c_{\min } / c_{\max }$, where $c_{\min } / c_{\max }$ is the minimum/maximum value of the concentration near the interface of the primary tip at the same time. The minimum concentration keeps decreasing with larger heat flux, while the maximum concentration keeps increasing; thus, the solute diffusion coefficient shows an increasing trend with increasing heat flux. The solute diffusion coefficient $k_{c}$ is equal to the solute segregation coefficient $k_{v}=c_{\text {solid }} / c_{\text {liquid }}$ in the solid-liquid interface near the dendrite tip, where $c_{\text {solid }} / c_{\text {liquid }}$ represents the equilibrium concentration at the interface. As shown in Figure 11, the large undercooling resulting from the heat extraction drives $k_{v}$ toward 1 during rapid solidification which is confirmed with the approximate analytic solution about the rapid dendrite growth in undercooled melts [30].

Therefore, heat flux induced from boundaries can significantly affect the dendrite growth in the undercooled melt. As shown in Figure 12, the undercooling of the simulation zone is decreasing with enhanced heat flux input from the boundaries, which leads to the solid rate decreasing with large heat flux. Large heat extraction from the boundaries can enhance the dendrite microstructure formation with strong side branches, while large heat input at the boundaries prevents the dendrite growth as well as secondary arm formations. Similar results have been observed and reported in many experimental studies in recent years [31-35].

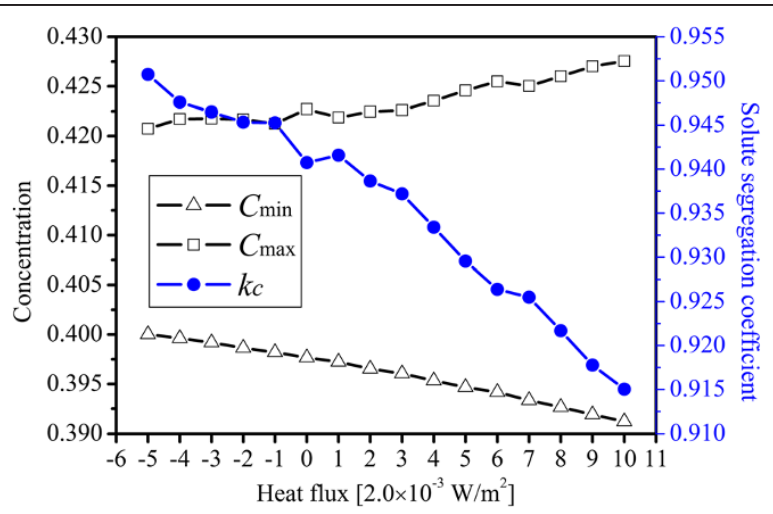

Figure 11 Effect of different heat fluxes on the solute diffusion coefficient. 


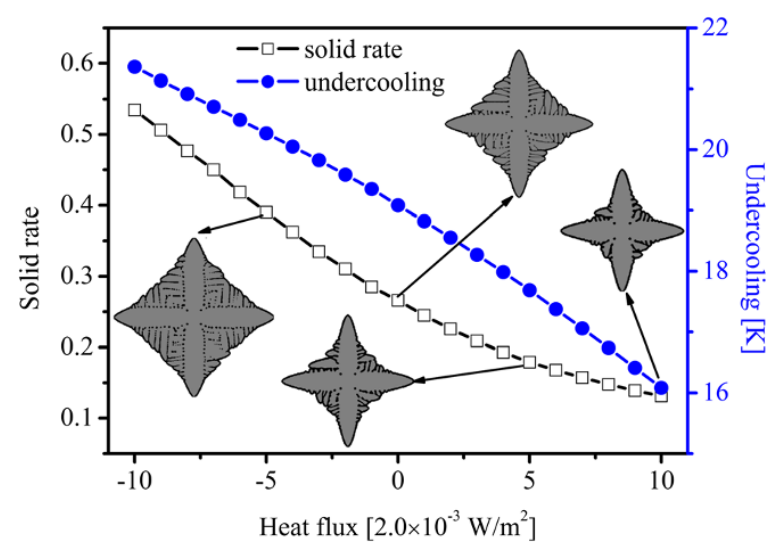

Figure 12 Effect of different heat fluxes on undercooling change and the solid rate during dendrite formations.

\section{Conclusions}

A phase field model for simulating solidification in binary alloys under non-isothermal conditions is implemented to study the effect of heat flux input/extraction from boundaries on the dendrite structure forming process in a $\mathrm{Ni}-\mathrm{Cu}$ alloy. In order to suppress the solute trapping due to the larger interface width used in simulations, the antitrapping current is introduced into the diffusion equation. Simulations were carried out to study the dendrite structure formation, concentration, and temperature changes with different boundary heat fluxes. It is obvious that heat flux input can raise the temperature near the boundary and results in the prevention of dendrite arm growth and side branching, while heat flux extraction from the boundaries can enhance the dendrite arm growth and secondary arm formations. The heat flux can also lead to the concentration distribution changes by changing the temperature distribution. High temperature can enlarge the solute segregation and decrease the solute level inside the dendrite structure decrease. The effect of different heat fluxes on solute segregation and concentration distributions inside dendrite structures is studied. With heat flux changing from large extraction to large input, the dendrite tip speed of the primary arm shows different changes, and the average secondary dendrite arm spacing decreases. Heat extraction from the boundaries could partly counterbalance latent heat release from solidification and lead to uniform concentration distributions in solid, while heat input, coupling with latent heat, would significantly raise temperatures at interfaces and decreases concentration with time evolution. These effects could be enlarged with increasing heat flux. Therefore, the heat flux input/extraction from the boundary can significantly change the morphology, concentration, and temperature distribution of the dendrite growth in undercooled melts, and these morphology and concentration changes will affect the properties of the alloys.

\section{Availaiblity of supporting data}

The computer code to reproduce all of the case studies presented in this manuscript is available upon request. 


\section{Competing interests}

The authors declare that they have no competing interests.

\section{Authors' contributions}

LD did all calculations and prepared the first version of the article. RZ discussed the simulation results and corrected the article. Both authors read and approved the final manuscript.

\section{Authors' information}

LD is a Ph.D. Candidate in the Department of Applied Physics in Northwestern Polytechnical University, whose research interest is phase-field simulation of solidification in metal alloys under different conditions. RZ is a Professor in the Department of Applied Physics in Northwestern Polytechnical University, whose research interest is solidifying process under mutiphysics fields.

\section{Acknowledgements}

The authors would like to thank the financial support from the NPU Foundation of Fundamental Research, China (No. JC201272).

Received: 7 February 2014 Accepted: 29 April 2014

Published: 28 June 2014

\section{References}

1. Asta M, Beckermann C, Karma A, Kurz W, Napolitano R, Plapp M, Purdy G, Rappaz M, Trivedi R (2009) Solidification microstructures and solid-state parallels: recent developments, future directions. Acta Mater 57:941-971

2. Chen LQ (2002) Phase-field models for microstructure evolution. Annu Rev Mater Res 32:113-140

3. Steinbach I (2009) Phase-field models in materials science. Model Simul Mater Sc 17:073001

4. Singer-Loginova I, Singer HM (2008) The phase field technique for modeling multiphase materials. Rep Prog Phys 71:106501

5. Boettinger WJ, Warren JA, Beckermann C, Karma A (2002) Phase-field simulation of solidification. Annu Rev Mater Res 32:163-194

6. Kobayashi R (1993) Modeling and numerical simulations of dendritic crystal-growth. Physica D 63:410-423

7. Wheeler AA, Boettinger WJ, Mcfadden GB (1992) Phase-field model for isothermal phase-transitions in binary-alloys. Phys Rev A 45:7424-7439

8. Wheeler AA, Boettinger WJ, Mcfadden GB (1993) Phase-field model of solute trapping during solidification. Phys Rev E 47:1893-1909

9. Kim SG, Kim WT, Suzuki T (1999) Phase-field model for binary alloys. Phys Rev E 60:7186-7197

10. Kim SG, Kim WT, Suzuki T (1998) Interfacial compositions of solid and liquid in a phase-field model with finite interface thickness for isothermal solidification in binary alloys. Phys Rev E 58:3316-3323

11. Karma A (2001) Phase-field formulation for quantitative modeling of alloy solidification. Phys Rev Lett 87:045501

12. Karma A, Rappel WJ (1998) Quantitative phase-field modeling of dendritic growth in two and three dimensions. Phys Rev E 57:4323-4349

13. Tong X, Beckermann C, Karma A, Li Q (2001) Phase-field simulations of dendritic crystal growth in a forced flow. Phys Rev E 63:061601

14. Jeong JH, Goldenfeld N, Dantzig JA (2001) Phase field model for three-dimensional dendritic growth with fluid flow. Phys Rev E 64:041602

15. Tsai YL, Chen CC, Lan CW (2010) Three-dimensional adaptive phase field modeling of directional solidification of a binary alloy: 2D-3D transitions. Int J Heat Mass Tran 53:2272-2283

16. Du LF, Zhang R, Zhang LM (2013) Phase-field simulation of dendritic growth in a forced liquid metal flow coupling with boundary heat flux. SCIENCE CHINA Technological Sciences 56:2586-2593

17. Nestler B, Choudhury A (2011) Phase-field modeling of multi-component systems. Curr Opin Solid St M 15:93-105

18. Nestler B, Garcke H, Stinner B (2005) Multicomponent alloy solidification: phase-field modeling and simulations. Phys Rev E 71:041609

19. Zhang RJ, Li M, Allison J (2010) Phase-field study for the influence of solute interactions on solidification process in multicomponent alloys. Comp Mater Sci 47:832-838

20. Feest EA, Doherty RD (1973) Dendritic solidification of Cu-Ni alloys. 2. Influence of initial dendrite growth temperature on microsegregation. Metall Trans 4:125-136

21. Viskanta R (1988) Heat-transfer during melting and solidification of metals. J Heat Trans-T Asme 110:1205-1219

22. Kumar TSP, Prabhu KN (1991) Heat-flux transients at the casting chill interface during solidification of aluminum base alloys. Metall Trans B 22:717-727

23. Juric D, Tryggvason G (1996) A front-tracking method for dendritic solidification. J Comput Phys 123:127-148

24. Amberg G, Tonhardt R, Winkler C (1999) Finite element simulations using symbolic computing. Math Comput Simulat 49:257-274

25. Tang JJ, Xue XA (2009) Phase-field simulation of directional solidification of a binary alloy under different boundary heat flux conditions. J Mater Sci 44:745-753

26. Loginova I, Amberg G, Agren J (2001) Phase-field simulations of non-isothermal binary alloy solidification. Acta Mater 49:573-581

27. Warren JA, Boettinger WJ (1995) Prediction of dendritic growth and microsegregation patterns in a binary alloy using the phase-field method. Acta Metall Mater 43:689-703 
28. Lan CW, Shih CJ (2004) Phase field simulation of non-isothermal free dendritic growth of a binary alloy in a forced flow. J Cryst Growth 264:472-482

29. Xu JJ, Wang TM, Zhu J, Xie HL, Xiao TQ, Li TJ (2011) In situ study on secondary dendrite arm coarsening of Sn-Bi alloy by synchrotron microradiography. Mater Res Innov 15:156-159

30. Kurz WFWJ (1998) Fundamentals of Solidification. Trans Tech Publications, Switzerland

31. Herlach D (2011) Crystal nucleation and dendrite growth of metastable phases in undercooled melts. J Alloy Compd 509:513-S17

32. Chang J, Wang HP, Zhou K, Wei B (2012) Rapid dendritic growth and solute trapping within undercooled ternary $\mathrm{Ni}-5 \% \mathrm{Cu}-5 \% \mathrm{Mo}$ alloy. Appl Phys A-Mater 109:139-143

33. Cao CD, Wang F, Duan LB, Bai XJ (2011) Effect of solidification temperature range on the dendritic growth mode. Sci China Phys Mech 54:89-94

34. Song RB, Dai FP, Wei BB (2011) Dendritic growth and solute trapping in rapidly solidified Cu-based alloys. Sci China Phys Mech 54:901-908

35. Yang XB, Fujiwara K, Maeda K, Nozawa J, Koizumi H, Uda S (2011) Dependence of Si faceted dendrite growth velocity on undercooling. Appl Phys Lett 98:012113

doi:10.1186/s40192-014-0018-4

Cite this article as: Du and Zhang: Phase field simulation of dendrite growth with boundary heat flux. Integrating Materials and Manufacturing Innovation 2014 3:18.

Submit your manuscript to a SpringerOpen ${ }^{\circ}$ journal and benefit from:

- Convenient online submission

- Rigorous peer review

- Immediate publication on acceptance

Open access: articles freely available online

- High visibility within the field

- Retaining the copyright to your article

Submit your next manuscript at $\gg$ springeropen.com 